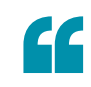

\section{the TNF-}

TNFR1

pathway triggered two types of RIPK1mediated cell death in the absence of IKK2

EXPERIMENTAL ARTHRITIS

\title{
TNF chronicity modifies synovial cell death
}

Inhibition of IאB kinase 2 (IKK2, also known as IKK $\beta$ ) in synovial fibroblasts can have differing effects on TNF-induced inflammatory arthritis, depending on the chronicity of TNF signals, according to new findings. "We identified a novel role of IKK2 in synovial fibroblasts in promoting ... chronic TNF-mediated joint destruction while prohibiting ... [receptor-interacting serine/ threonine-protein kinase 3 (RIPK3)]mediated inflammation in the joint, indicating that chronic inflammatory conditions might modify the predicted effects of a druggable target," reports co-corresponding author Marietta Armaka.

TNF is a key inflammatory mediator in rheumatoid arthritis (RA). Multiple pathways are activated downstream of TNF receptor superfamily member 1A (TNFR1, also known as p55) that lead to survival and inflammatory signals, as well as cell death pathways, the balance of which is highly dependent on nuclear factor- $\kappa \mathrm{B}(\mathrm{NF}-\kappa \mathrm{B})$ activation. Although inhibition of the NF- $\kappa B$ pathway can effectively downregulate immune cell activation, less is known about the effects of inhibiting this pathway on synovial fibroblasts or on cell death.

Mesenchymal-specific knockout of TNFR1 prevented disease development in three mouse models of either acute or chronic TNF-induced arthritis. Furthermore, mesenchymalspecific deletion of IKK2 similarly protected mice from bone erosions and cartilage degradation in acute or chronic TNF-induced arthritis, but did not inhibit synovitis.

"The loss of IKK2 signalling specifically in synovial fibroblasts led to the induction of death in these cells in the synovium," reports Armaka. The investigators showed that in synovial fibroblasts, the TNF-TNFR1 pathway triggered two types of RIPK1-mediated cell death in the absence of IKK2: a caspase-dependent pathway, leading to apoptosis (observed mostly in acute arthritis) and a RIPK3mediated, caspase-independent pathway, leading to necroptosis and contributing to the inflammatory phase in chronic arthritis.

"We believe that the detection of different [cell] death pathways
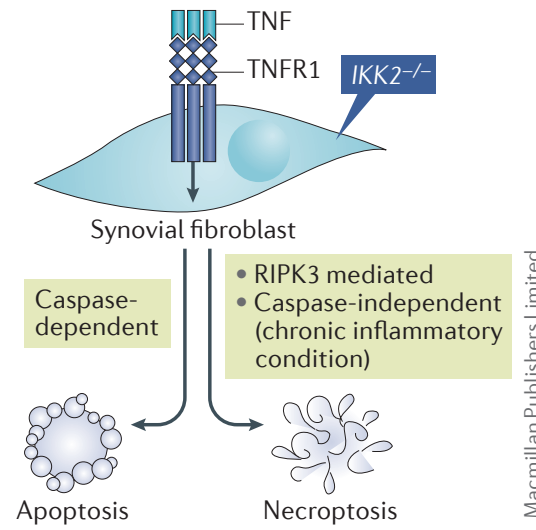

in [synovial fibroblasts] reflects the heterogeneity of responses within the [synovial fibroblast] population," says co-corresponding author George Kollias. "By analyzing these differential responses, we hope that in future it will be possible to suppress the pathogenic [synovial fibroblasts] by combined targeting of survival and [cell] death pathways."

Jessica McHugh

ORIGINAL ARTICLE Armaka, M. et al. The p55TNFR-IKK2-Ripk3 axis orchestrates arthritis by regulating death and inflammatory pathways in synovial fibroblasts. Nat. Commun. 9, 618 (2018) 